\title{
Performance Analysis of IEEE 802.11 in Multi-hop Wireless Networks
}

\author{
Lan Tien Nguyen ${ }^{1}$, Razvan Beuran ${ }^{2,1}$, Yoichi Shinoda ${ }^{1,2}$ \\ ${ }^{1}$ Japan Advanced Institute of Science and Technology, \\ 1-1 Asahidai, Nomi, Ishikawa, 923-1292 Japan \\ ${ }^{2}$ National Institute of Information and Communication Technology \\ Hokuriku Research Center, 2-12 Asahidai, Nomi, Ishikawa, 923-1211 Japan \\ lannt@jaist.ac.jp
}

\begin{abstract}
Multi-hop wireless networks provide a quick and easy way for networking when we need a temporary network or when cabling is difficult. The 802.11 Medium Access Control (MAC) plays an important role in the achievable system performance. There have been many studies on analytic modeling of single-hop 802.11 wireless networks but only a few on the analysis of multihop wireless networks. Furthermore, the object of these researches is an homogeneous ad-hoc wireless networks; therefore they are not appropriate for a network with structure such as wireless mesh networks. This paper introduces an analytic model of throughput performance for the IEEE 802.11 multi-hop networks, which allows us to compute the achievable throughput on a given path in multi-hop wireless networks. The model shows that there is an optimal point at which throughput is maximized. Using this model and a Markov model for modeling the operation of the IEEE 802.11 DCF we can determine the amount of data that each node should inject to the network to get the best throughput performance.
\end{abstract}

\section{Introduction}

Multi-hop wireless networks provide a quick and easy way for networking when we need a temporary network or when cabling is difficult. The 802.11 Distributed Coordination Function, Carrier Sense Multiple Access with Collision Avoidance (CSMA/CA) based, is the most popular MAC protocol for wireless communication. The analysis in this paper is based on the operation of 802.11 DCF.

In wireless networks, the throughput that can be sent through a wireless link depends on various factors such as the distance between nodes, the transmission power, characteristics of environment like path loss, fading, noise, etc. Given the broadcast nature in wireless environment, the achievable throughput on a wireless link not only dependent on the operation data rate on that link, but also depends on number of nodes using the same radio channel within the two end-points carrier sense area. Considering a traffic flow sent from source node to destination node through other intermediate nodes, the middle nodes may have to contend with more nodes than the source node or the destination node does. Consequently, the source node can inject more data into the path than the amount that can be forwarded by middle nodes. This 
may lead to packet loss and low performance in wireless network since the cost to resend a packet is high and when injecting the amount of data larger than the one the channel can accepted makes the condition even worse and leads to a high packet loss. High packet loss in turn can trigger re-routing and make the network topology instable [1].

To date, there have been a lot of studies on analytic modeling of both single-hop 802.11 wireless networks [2], [3], [4], [5], [6] and multi-hop wireless networks [7], [8], [9] [10]. All of these models are assumed to use saturated traffic load (which mean a node always has a packet ready for transmission) except the model which is proposed in [10]. Other studies focus on the theoretical upper bound of throughput on an homogeneous ad-hoc network [11], [12] or are based on the assumption of global scheduling [13], [14], [15], which may not be a good assumption in a real wireless networks using IEEE 802.11.

In this paper, we introduce an analytic model of throughput performance for 802.11 multi-hop networks allowing us to compute the achievable throughput on a given path in multi-hop wireless networks. The model shows that there is an optimal point at that throughput is maximized. Based on this model and Markov model for modeling the operation of the IEEE 802.11 DCF we can determine the amount of data that each node should inject to the network to get the best throughput performance.

\section{Related work}

Following the analytical methodology introduced for the analysis of ALOHA protocol and carrier sense multiple access [16], [17], a lot of analytical modeling of wireless MAC protocols has often focused on single-hop wireless networks. Although some models are proposed for multi-hop wireless networks [18], [19], they can't model the effect of binary exponential back-off scheme (BEB) which is the key to adjust transmission intervals in IEEE 802.11. Recently there are more researches on modeling IEEE 802.11 DCF in single-hop wireless networks [2],[3], [4], [5], [6]. With a Markov Chain model, the exponential back-off scheme was accurately modeled. However, these models can't be directly applied to multi-hop wireless networks due to the hidden node problem.

One of the first analytical models of IEEE 802.11 DCF for multi-hop wireless networks is proposed in [7]. The hidden node problem is taken into account, but transmission of all the nodes is assumed to follow a Poisson process which doesn't match the behavior of IEEE 802.11 binary exponential back-off scheme. In the model which is proposed by Wang and Garcia-Luna-Aceves in [8] the BEB scheme can't be captured effectively either because only a simple model for exponential back-off scheme is used.

Some recent works have solved the problem to model behavior of binary exponential back-off scheme [9], [10]. Carvalho and Garcia-Luna-Aceves introduced an analytical model to study operation of 802.11 DCF in multi-hop wireless networks [9]. The model takes into account the impact of both physical layer and network topology. However, the impact of hidden nodes is not considered. David Malone et al. proposed a model which is effective in capturing the binary exponential back-off scheme of 
802.11 DCF in both saturated and non-saturated environment [10]. This model helps us in studying the relation between input load and output load of a node depending on several parameters of IEEE 802.11 DCF.

There are some research results on the capacity of general ad-hoc network [11], [12] and mesh network [13]. It was shown that for stationary networks, the capacity for each node decrease as $\mathrm{O}(1 / \sqrt{n})$; meanwhile for the mobile networks that can tolerate long delay, the capacity may remain constant. In mesh networks, the authors in [13] claimed that gateways are bottlenecks and the available capacity for each node reduces to $\mathrm{O}(1 / n)$, where $n$ is the number of node associated with one gateway.

\section{Network model}

We consider a multi-hop wireless network in which each node uses IEEE 802.11 DCF for medium access control and has only one radio interface operating on the same radio channel. In our model we also assume that only one transmission rate is used although $802.11 \mathrm{a} / \mathrm{b} / \mathrm{g}$ standards support multiple transmission rates

At the MAC layer, the data payload is assumed to be 1024 bytes plus 34 bytes from MAC header. Request-to-Send (RTS) and Clear-to-Send (CTS) are assumed to be sent at the lowest data rate that is supported by physical layer.

At the application layer, we assume that there is single data flow and our goal is to present an analytical model to compute achievable throughput along a path. Because of having only one flow in the network so the model we use only consider intra-flow interference occurring for packets of the same flow transported over different wireless links. The inter-flow will be taken into account in our future model.

\section{Throughput Analysis}

The analytical model that we use to compute achievable throughput along a wireless multi-hop path is based on the model presented in [2] but it takes into account hidden nodes which strongly affects achievable throughput in multi-hop wireless networks.

Similar to [11] we define three radio ranges:

Transmission range $\left(\boldsymbol{R}_{t c}\right)$ : represents the range in which a frame can be successfully received if there is no interference from other nodes. This value is determined by transmission power, receiver sensitivity and radio propagation properties. A node B is considered in transmission range of node $\mathrm{A}$ if packets come from node $\mathrm{A}$ are received at node $\mathrm{B}$ with power higher than minimum reception power of node $\mathrm{B}$.

Carrier sense range $\left(\boldsymbol{R}_{c s}\right)$ : represents the range in which a transmission can trigger carrier sense detection at radio interface of the node. This value is determined by receiver's sensitivity and also transmission power, radio propagation properties. A node $\mathrm{B}$ is considered in carrier sense range of node $\mathrm{A}$ if packets come from node $\mathrm{A}$ are received at node $\mathrm{B}$ with power higher than minimum detection power of node $\mathrm{B}$.

Interference range $\left(\boldsymbol{R}_{\boldsymbol{i}}\right)$ : represents the range in which the station in receive mode will be interfered by other transmitter and thus suffers a loss. 
Considering a transmission from node $\mathrm{A}$ to node $\mathrm{B}$, the hidden nodes in this case are the nodes that are inside the interference range of node B (receiver) but outside both transmission range of node $\mathrm{B}$ and carrier sense range of node $\mathrm{A}$ (transmitter). Let's say $\mathrm{C}$ is a node in the hidden node set. Because $\mathrm{C}$ is outside the carrier sense range of node $\mathrm{A}$ so it is not aware of transmissions between $\mathrm{A}$ and $\mathrm{B}$. Any transmission of $\mathrm{C}$ will corrupt the transmission between A and B. The RTS/CTS scheme can't solve hidden node problem, and this reason makes performance of IEEE 802.11 worse in multi-hop wireless networks. Our model will take into account the impact of the hidden node problem on the throughput of a given path.

We consider $n$ fixed nodes and $n-1$ nodes send traffic to the next nodes along that path as illustrated in the Figure 1. The following notations are used to denote subset of $n$ nodes.

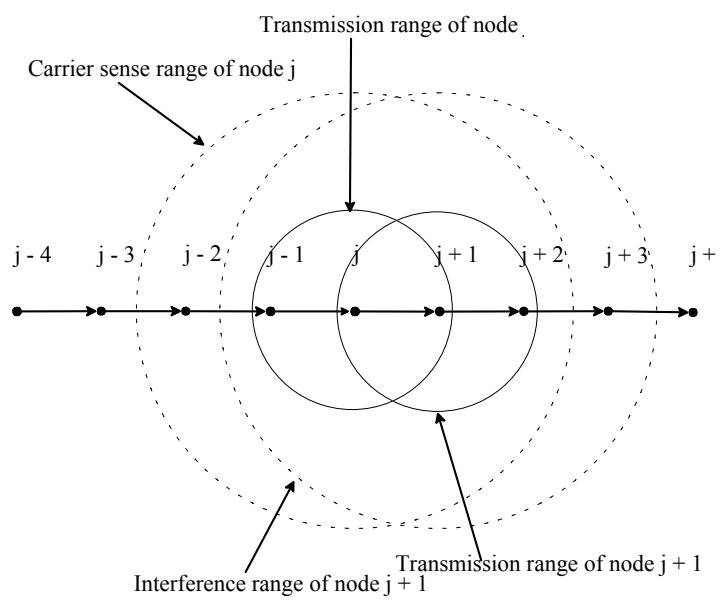

Fig. 1. Illustration of the three different ranges.

- $\quad C_{j}$ refers to subset of nodes within carrier sense range of node $j$.

- $C_{j}^{+}$refers to the subset $C_{j}$ plus node $j$ itself.

- $\quad I_{j}$ refers to the subset of nodes within interference range of node $j$.

- $\quad T_{j}$ refers to the subset of node within transmission range of node $j$.

Let $S$ to be normalized system throughput, which can be compute as fraction of time that channel is used to successfully transmit payload data. In a slot time channel can be idle, busy with a successful transmission or busy with a collision. In order to calculate the average length of a slot time we have to consider what can happen in a slot time or the probability that channel has one of three different states. Let $n$ be maximum number of nodes in the interference range of nodes in the path, $\tau$ the probability that a node transmits in a random chosen slot time, and $P_{t r}$ the probability that there is at least one transmission in the considered slot time. One time slot is denoted as $\sigma$. Because of $n$ different nodes content on the channel, each node will transmit in a slot time with probability $\tau$.

$$
P_{t r}=1-(1-\tau)^{n}
$$


Consider a transmission between node $j$ and node $j+1$ and successful transmission need $k$ time slot. Let $P_{s}$ be the probability that the transmission is successful, which is given by probability that there is only the sender transmit at the time in $C_{j}$ and none of nodes in $\left\{I_{j} U I_{j+1}\right\} /\left\{T_{j} U T_{j+1}\right\}$ transmit in $k$ time slot. Let $h$ be number of nodes in $\left\{I_{j} U I_{j+l}\right\} /\left\{T_{j} U T_{j+1}\right\}$, these nodes are called hidden nodes.

$$
P_{s}=\frac{n \tau(1-\tau)^{n-1}(1-\tau)^{h k}}{P_{t r}}
$$

We can express the achievable throughput $S$ as the following ratio

$$
S=\frac{E_{p}}{E_{s l}}
$$

Where $E_{p}$ is average amount of payload information is successfully transmitted in a slot time, $E_{s l}$ being average length of a slot time.
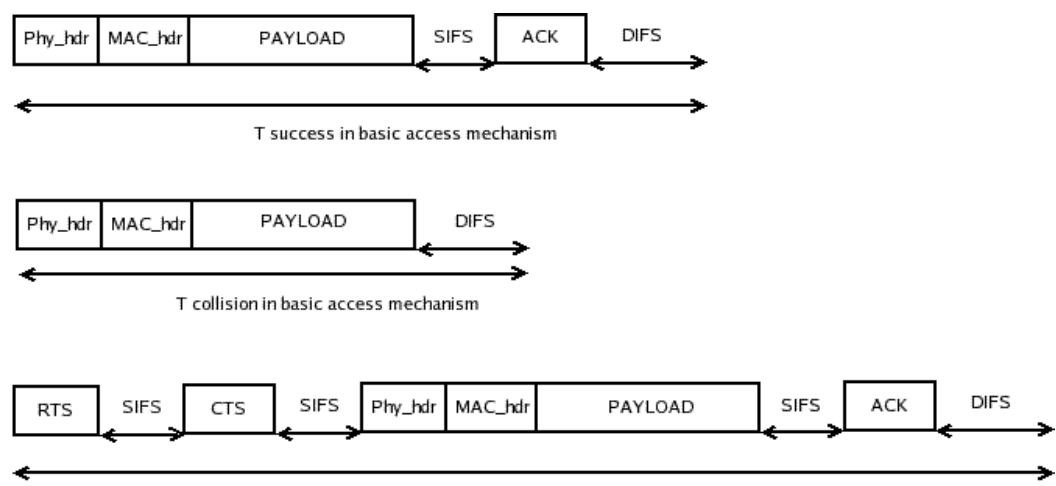

T success in RTS/CTS mechanism

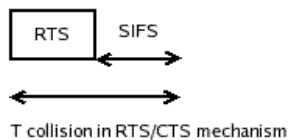

Fig. 2. Success time and collision time with basic access and RTS/CTS mechanism.

Let $E(P)$ be average packet payload size. Since a packet can be transmitted successfully in a slot time with probability $P_{t r} \cdot P_{s}$, we may have

$$
E_{p}=P_{t r} P_{s} E(P)
$$

The average length of a slot time is obtained considering that: a slot time is idle with probability $1-P_{t r}$; contain a successful transmission with probability $P_{t r} P_{s}$ and contains a collision with probability $P_{t r}\left(1-P_{s}\right)$. Thus equation (3) can be expressed as

$$
S=\frac{(1 / n) P_{t r} P_{s} E[P]}{\left(1-P_{t r}\right) \sigma+P_{t r} P_{s} T_{s}+P_{t r}\left(1-P_{s}\right) T_{c}}
$$


Where $T_{s}$ is the average time that the channel is sensed busy by a successful transmission, $T_{c}$ being average time that channel is sensed busy by a collision and $\sigma$ is duration of a slot time. The value of $T_{s}$ and $T_{c}$ depends on the access mechanism used.

Firstly, we consider the system uses the basic access mechanism. Denoting $\delta$ as propagation delay, from Figure 2 we can obtain the value of $T_{s}^{b a s}$ and $T_{c}^{\text {bas }}$ as follows

$$
\begin{gathered}
T_{s}^{\text {bas }}=P h y_{-} h d r+M A C_{-} h d r+E[P]+S I F S+\delta+A C K+D I F S+\delta \\
T_{c}^{\text {bas }}=P h y_{-} h d r+M A C_{-} h d r+E\left[P^{*}\right]+D I F S+\delta
\end{gathered}
$$

where $E\left[P^{*}\right]$ is the average length of longest packet involve in the collision. In our model we assume that all packets have the same payload length so that $E\left[P^{*}\right]=E[P]$ $=P$

Secondly, considering the system in which each packet is transmitted by using RTS/CTS mechanism $T_{s}$ and $T_{c}$ can be computed by the following equations:

$$
\begin{gathered}
T_{s}^{R T S}=R T S+S I F S+\delta+C T S+S I F S+\delta+P h y_{-} h d r \\
+M A C_{-} h d r+E[P]+S I F S+\delta+A C K+D I F S+\delta \\
T_{c}^{R T S}=R T S+D I F S+\delta
\end{gathered}
$$

In order to see the relation between $S$ and $\tau$ we rearrange equation (5) as

$$
S=\frac{(1 / n) E[P]}{T_{s}-T_{c}+\frac{\sigma}{P_{t r} P_{s}}\left[P_{t r}\left(T_{c}^{*}-1\right)+1\right]}
$$

where $T_{c}^{*}=\frac{T_{c}}{\sigma}$.

As $T_{s}, T_{c}, n, E[P]$ and $\sigma$ are constants and let us call

$$
F(\tau)=\frac{1}{P_{t r} P_{s}}\left[P_{t r}\left(T_{c}^{*}-1\right)+1\right]
$$

From (1) (2) and (11)

$$
F(\tau)=\frac{1}{n}\left[\frac{T_{c}^{*}}{\tau(1-\tau)^{n+h k-1}}-\frac{T_{c}^{*}-1}{\tau(1-\tau)^{h k-1}}\right]
$$

By analyzing the relation between $F$ and $\tau$ we can get the relation between $S$ and $\tau$. Taking the derivative of (12) with respect to $\tau$.

$$
\frac{d F}{d \tau}=\frac{\left(T_{c}^{*}-1\right)(1-\tau)^{n}+T_{c}^{*} \tau(n+h k)-T_{c}^{*}}{n \tau^{2}(1-\tau)^{n+h k}}
$$

It is easy to see that 


$$
\begin{aligned}
& \lim _{\tau \rightarrow 0^{+}} \frac{d F}{d \tau}<0 \\
& \lim _{\tau \rightarrow 1^{-}} \frac{d F}{d \tau}>0
\end{aligned}
$$

From (14) and (15) there must be a value of $\tau$ in the range [0:1] at that the value of $F$ is minimum and hence it maximizes the value of $S$. It is possible to assume that $\tau \ll$ 1. That assumption comes from the mechanism of the IEEE 802.11 DCF standards [12]. According to [12] the minimum value of contention window $\left(C W_{0}\right)$ is 32 so the value of $\tau$ should be smaller than $1 / C W_{0}(0.0321)$ and our assumption is valid. To find that value of $\tau$ under the condition $\tau \ll 1$ we can approximate

$$
(1-\tau)^{n} \approx 1-n \tau+\frac{n(n-1)}{2} \tau^{2}
$$

Making equation $\frac{d F}{d \tau}=0$ to be quadratic equation and then we can solve it.

Table 1. System Parameters.

\begin{tabular}{|l|l|}
\hline Packet Payload & 1024 bytes \\
\hline MAC header & 272 bits \\
\hline Physical header & 192 bits \\
\hline ACK & 112 bits + Physical header \\
\hline RTS & 160 bits + Physical header \\
\hline CTS & 112 bits + Physical header \\
\hline Channel Bit Rate & $1 \mathrm{Mbit} / \mathrm{s}$ \\
\hline Propagation delay & $1 \mu \mathrm{s}$ \\
\hline Slot Time & $20 \mu \mathrm{s}$ \\
\hline SIFS & $10 \mu \mathrm{s}$ \\
\hline DIFS & $50 \mu \mathrm{s}$ \\
\hline
\end{tabular}

Figure 6 shows the theoretical maximum throughput that can achieve with DCF with the RTS/CTS mechanism. The parameters used to compute numerical results are summarized in Table 1.

\section{Simulation and Analysis Results}

In this section some results of both analysis and simulation are presented for evaluating the effectiveness of the proposed analytical model. For simulation we use ns-2.27 [22], with CMU Monarch Project wireless and mobile ns-2 extensions [23]. The network topology used includes 100 nodes, which are putted in a line. The distance between two nodes is changed from $200 \mathrm{~m}$ to $54 \mathrm{~m}$ to vary the number of nodes in the 
interference range. The transmission range and carrier sense range are set to be $250 \mathrm{~m}$ and $550 \mathrm{~m}$, respectively. The data rate used in all simulations is $1 \mathrm{Mbps}$ and the other parameters are set according to the Table 1. The traffic source for a node will send data at several constant rates; these rates are set to one of the following rate 20,30, $40,50,70,90,120,150,200 \mathrm{Kbps}$ for monitoring the changes of throughput at each node.

The simulation results in Figure 3, 4 and 5 show that when the input traffic load increases 802.11 multi-hop wireless network will get to saturation status but with higher number of node in the interference range the throughput will be decreased quickly because of collisions.

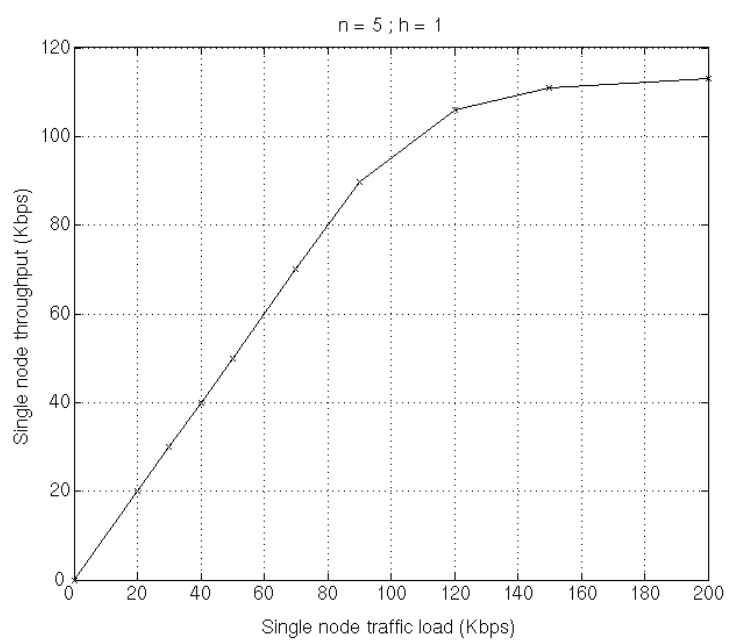

Fig. 3. Single node throughput versus single node traffic load $(n=5 ; h=1)$.

In the analytical model, the input traffic load is represented by transmission probability $\tau$, where $\tau$ is the probability that a node transmits in a random chosen slot time. The value of $\tau$ obviously depends on the node's input traffic load, traffic of other nodes in its carrier sense range and the mechanism is used to access media (IEEE 802.11 DCF in this case). By using a Markov model, the relation between $\tau$ and input traffic load can be obtain from a research of K. Duffy et al [10]. 


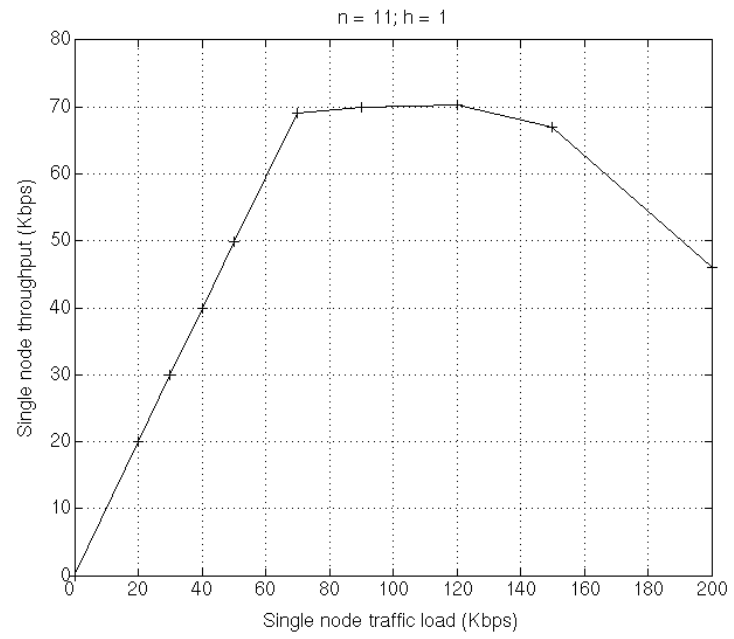

Fig. 4. Single node throughput versus single node traffic load $(n=11 ; h=1)$.

$$
\tau=\frac{1}{\eta}\left(\frac{q^{2} W_{0}}{(1-q)(1-p)\left(1-(1-q)^{W_{0}}\right)}-\frac{q^{2}(1-p)}{1-q}\right)
$$

where

$$
\begin{aligned}
& \eta=\frac{q W_{0}}{1-(1-q)^{W_{0}}}+\frac{q W_{0}\left(q W_{0}+3 q-2\right)}{2(1-q)\left(1-(1-q)^{W_{0}}\right)} \\
& +(1-q)+\frac{q\left(W_{0}+1\right)\left(p(1-q)-q(1-p)^{2}\right)}{2(1-q)} \\
& +\frac{p q^{2}}{2(1-q)(1-p)}\left(\frac{W_{0}}{1-(1-q)^{W_{0}}}-(1-p)^{2}\right) \\
& \left(\frac{2 W_{0}\left(1-p-p(2 p)^{M-1}\right)}{(1-2 p)}+1\right)
\end{aligned}
$$

Here, $p$ is the probability that a node senses the channel busy on an attempted transmission, $q$ being the probability that the node's buffer has packets waiting for transmission, $W_{0}$ being the minimum contention window of the node and $W_{0}^{M}$ being the node's maximum contention window size. $M$ is the maximum back-off stage.

On other hand, based on the nature of wireless environment we have a relation between $\tau$ and $p$

$$
1-p=(1-\tau)^{n-1}
$$

where $n$ is number of node in the interference range of the node including itself. Based on the assumption that we can compute the value of $q$ from given input traffic load of the node, by solving equations (17) and (18) we can find the value of $p$ and $\tau$ 
at that the node operates. Getting the value of $\tau$ by doing so is somehow difficult. However, with the given network topology, the analytical model can predict the maximum throughput of a node or the input traffic load can be used to saturate the network.

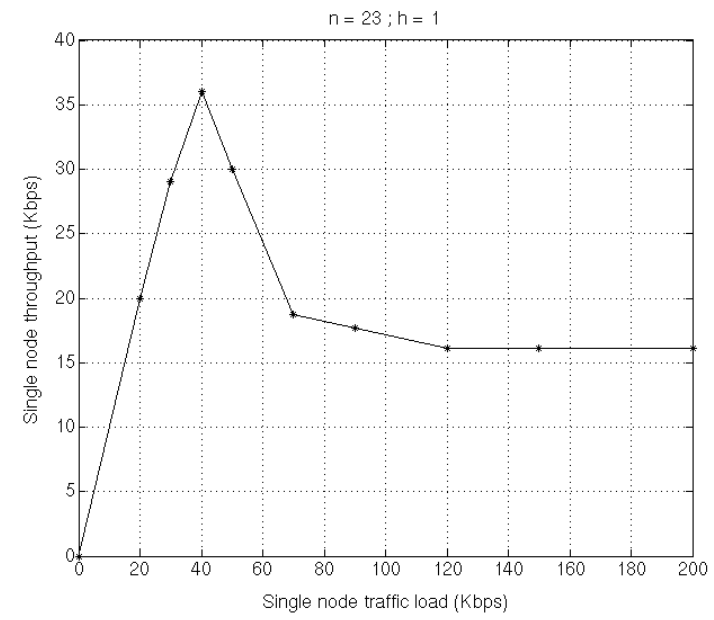

Fig. 5. . Single node throughput versus single node traffic load $(n=23 ; h=1)$.

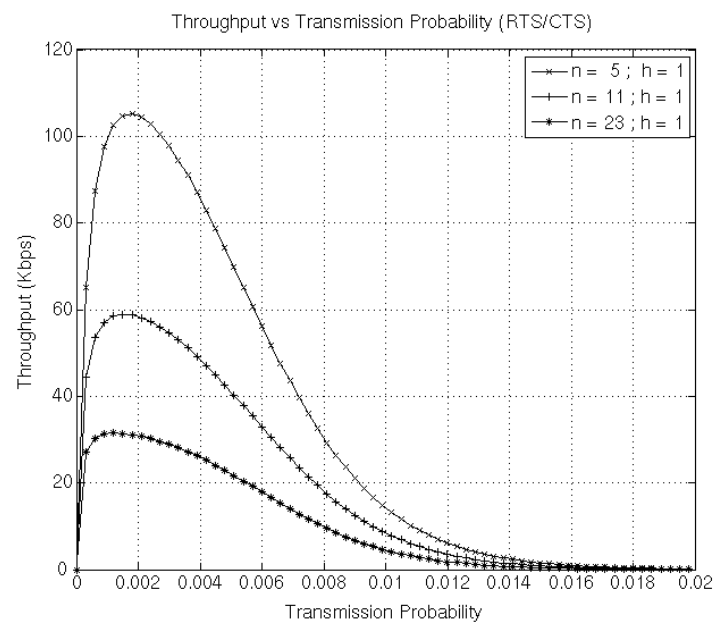

Fig. 6. Single node throughput versus transmission probability $\tau$, obtained by the analytical model.

Figures 3, 4, and 5 present the simulation results with different network conditions while Figure 6 shows the analytical results. It is can be seen that the maximum values of throughput from simulation results are reasonably consistent with the maximum throughputs from analytical results. The maximum values of throughput in figures 3 , 4, and 5 are $114 \mathrm{kbps}, 70 \mathrm{kbps}, 36 \mathrm{kbps}$ while the analytical model gives us the values 
of $106 \mathrm{kbps}, 59 \mathrm{kbps}, 31 \mathrm{kbps}$ respectively. Thus we can say that the analytical model can be applied to predict the saturation throughput on a given path of 802.11 multihop wireless networks and consequently the optimum input traffic load is obtained.

\section{Conclusions}

In this paper we propose an analytical model for analyzing the throughput performance of IEEE 802.11 multihop wireless networks. Comparison with simulation results shows that the model is successful to estimate the saturation throughput on a given path in the multihop wireless network. The model also allows us to understand how the interference range and hidden node affect to throughput performance or say in other way the impact of physical conditions to MAC performance. By using this model performance of MAC protocol in IEEE 802.11 multihop wireless network can be studied more effectively. For the future work, we will study the issue of improving throughput performance in IEEE 802.11 wireless mesh network and in particular, we will focus on routing protocol and admission control mechanism for that network. We will also need a better model which allows us to predict per hop throughput as well as delay time and jitter with inter-flow interference and multiple transmission rates will be taken into account.

Acknowledgements. The financial support from the 21st century COE program "Verifiable and Evolvable e-Society", Japan Advanced Institute of Science and Technology, is gratefully acknowledged.

\section{References}

1. P. C. Ng, S. C. Liew, "Re-routing Instability in IEEE 802.11 Multi -hop Ad hoc Networks", IEEE WLN'04, Nov. 2004, Tampa, USA

2. G. Bianchi, "Performance analysis of the IEEE 802.11 distributed coordination function," IEEE Journal on Selected Areas in Communications, vol. 18, no. 3, pp. 535-547, 2000

3. Mohammad Hossein Manshaei, Gion Reto Cantieni, Chadi Barakat, Thierry Turletti: Performance Analysis of the IEEE 802.11 MAC and Physical Layer Protocol. WOWMOM 2005: 88-97

4. Chuan H. Foh, M. Zukerman, Performance Analysis of the IEEE 802.11 MAC Protocol, EW2002 Proceedings, 2002

5. Xiao Y and Rosdahl J, "Performance Analysis and Enhancement for the Current and Future IEEE 802.11 MAC Protocols," ACM SIGMOBILE Mobile Computing and Communications Review (MC2R), special issue on Wireless Home Networks, Vol. 7, No. 2, Apr. 2003, pp. 6-19

6. F. Cali, M. Conti, and E. Gregori. Dynamic tuning of the IEEE 802.11 protocol to achieve a theoretical throughput limit. IEEE/ACM Transactions on Networking, 8(6):785-799, December 2000

7. H. Chhaya and S. Gupta. Performance modeling of asynchronous data transfer methods of IEEE 802.11 MAC protocol. Wireless Networks, 3:217-234, 1997 
8. Y. Wang and J. J. Garcia-Luna-Aceves. Performance of collision avoidance protocols in single-channel ad hoc networks. In Proc. of ICNP, pages 184-190, November 2002

9. M. Carvalho and J. Aceves. Scalable model for channel access protocols in multihop ad hoc networks. In ACM Mobicom'04, September 2004

10.K. Duffy, D. Malone, and D. J. Leith, "Modeling the 802.11 Distributed Coordination Function in non-saturated conditions," IEEE Communications Letters, vol. 9, no. 8, pp. 715-717, 2005

11.P. Gupta, P. R. Kumar, "The Capacity of Wireless Networks", IEEE Trans. Inform. Theory, Vol.46, No.2, pp.388-404, Mar. 2000

12.J. Li, C. Blake et al., "Capacity of Ad Hoc Wireless Networks”, ACM MobiCom'01, Rome, Italy, July 2001

13.J. Jangeun and M. L. Sichitiu, "The nomial capacity of wireless mesh networks," IEEE Wireless Communications, pp. 8-14, Oct. 2003

14.K. Jain et al. "Impact of Interference on Multi-hop Wireless Network Performance", ACM MobiCom'03, San Diego, USA, Sept. 2003

15.M. Kodialam, T. Nandagopal, "Characterizing Achievable Rates in Multi-hop Wireless Networks: The Joint Routing and Scheduling Problem”, ACM MobiCom'03, San Diego, USA, Sept. 2003

16.L. Kleinrock and F. A. Tobagi. Packet switching in radio channels: Part I - carrier sense multiple-access modes and their throughput-delay characteristics. IEEE Transactions on Communications, 23(12):1400-1416, 1975

17.F. A. Tobagi and L. Kleinrock. Packet switching in radio channels: Part II - the hidden terminal problem in carrier sense multiple-access modes and the busy-tone solution. IEEE Transactions on Communications, 23(12):1417-1433, 1975

18. F. A. Tobagi. Analysis of a two-hop centralized packet radio network - part II: Carrier sense multiple access. IEEE Transactions on Communications, 28(2):208-216, February 1980

19.F. A. Tobagi. Analysis of a two-hop centralized packet radio network - part II: Carrier sense multiple access. IEEE Transactions on Communications, 28(2):208-216, February 1980

20.K. Xu, M. Gerla, and S. Bae, "How effective is the IEEE 802.11 RTS/CTS handshake in ad hoc networks," in Proc. of GLOBECOM '02, 2002

21.IEEE standards for wireless LAN medium access control (MAC) and physical layer (PHY) specifications. 1999

22.“The network simulator - ns2", http://www.isi.edu/nsnam/ns/

23.CMU Monarch Project Extensions to NS2, http://www.monarch.cs.cmu.edu/cmu-ns.html 